\title{
Ressources et utilisations de l'eau du bassin de la Loire Essai de comptabilité régionale de l'eau
}

\author{
P. Babillot (IFEN) \\ J. Margat (BRGM)
}

\section{I $⿴$ PREAMBULE}

L'idée d'établir des comptes de l'eau a été conçue dans le cadre des travaux de la commission interministérielle des comptes du patrimoine naturel, qui visait à promouvoir la prise en compte de l'environnement dans la Comptabilité Nationale, à partir de 1982 . Son objectif était de mettre en cohérence, suivant des références spatiales et temporelles homogènes et pertinentes, les estimations et les statistiques sur les ressources et les utilisations d'eau, afin de permettre une comptabilité analytique et une évaluation des pressions de celles-ci sur celles-là.

La première phase de ces travaux s'est attachée aux comptes en quantités simples et a seulement exploré les voies et les modes de comptes en qualité.

La CICPN a, à cette fin, adopté une classification, construit des tableaux comptables appropriés et défini une méthode pour traiter les données voulues. Les principes et les modalités de cette approche comptable ont été exposés dans la publication de l'INSEE sur les " Comptes du patrimoine naturel " [1], notamment dans son chapitre 5 "Comptes des eaux continentales".

Après un premier exercice de comptabilité régionale annuelle réalisé en 1983-84 sur le département du Calvados (divisé en 10 bassins élémentaires), pour éprouver la faisabilité de l'approche conçue, l'établissement de comptes annuels de la France entière et de chacun des six bassins (Agences de l'eau) pour l'année 1981, a été réalisé sous la responsabilité du ministère de l'Environnement (Direction de la prévention des pollutions/Service de l'eau), avec le concours du BRGM, en 1985. Les résultats du compte du bassin Loire-Bretagne entrepris dans ce cadre, sont présentés ci-après.

Plus récemment (1995), un nouvel exercice comptable a été réalisé à l'initiative de l'IFEN [2], pour actualiser l'évaluation de faisabilité de ces travaux dans un cadre plus régional - le haut bassin de la Loire - tout en affinant les références temporelles en opérant des comptes trimestriels dans le cadre de l'année hydrologique 1989-1990. Les résultats et les enseignements de ce second exercice, sur une partie du bassin de la Loire, sont présentés ensuite.

\section{1 LES COMPTES DE L'EAU : UNE APPROCHE ORIGINALE}

Par rapport aux chiffrages et aux statistiques classiques respectifs sur les ressources en eau et sur les utilisations opérées séparément, suivant des références territoriales et temporelles indépendantes et généralement sans cohérence, le "plus " apporté par l'approche comptable consiste à rapprocher des données d'origines très diverses, à unifier les références spatio-temporelles pour rendre les chiffrages cohérents, et à mettre en correspondance les subdivisions des systèmes pour permettre une comptabilité non seulement globale mais analytique.

A cette fin, la structure des tableaux comptables adoptés met en relation :

- Les subdivisions des systèmes de ressource en eau physiques en " branches" macroscopiques correspondant aux types de milieu - eau (eau du sol, eaux souterraines, cours d'eau, lacs...) qui constituent des catégories de source d'approvisionnement ;

- les opérations d'échanges spontanés entre ces sous-systèmes ou avec les agents utilisateurs (dans les deux sens);

Elle met aussi en correspondance les classes d'agents du système d'utilisation avec ces sous-systèmes de ressource (cf les principaux tableaux comptables 2 et 3 ).

Cette structure comptable impose de fortes contraintes de cohérence aux différents résultats chiffrés et elle permet le calcul de divers indicateurs d'économie de l'eau. Elle permet aussi en principe la liaison entre les comptes d'agent physiques et les comptes économiques monétaires.

\section{1 COMPTE DE L'EAU DU BASSIN LOIRE-BRETAGNE EN 1981}

Les deux principaux tableaux comptables établis, le " compte central " qui décrit l'ensemble des volumes d'eau annuels échangés dans le bassin et le " compte d'utilisation "qui décrit les quantités d'eau échangées par les agents entre eux et avec le milieu, sont présentés ci-après. 


\begin{tabular}{|c|c|c|c|c|c|c|}
\hline \multirow[b]{2}{*}{$\begin{array}{c}\text { Sources } \\
\text { d'approvision- } \\
\text { nement }\end{array}$} & \multicolumn{6}{|c|}{ Secteurs d'utilisation } \\
\hline & $\begin{array}{c}\text { Alimentation des } \\
\text { collectivités } \\
\text { (eau potable) }\end{array}$ & $\begin{array}{c}\text { Services publics } \\
\text { (voies navigables) }\end{array}$ & $\begin{array}{l}\text { Agriculture } \\
\text { (irrigation) }\end{array}$ & $\begin{array}{l}\text { Industries non } \\
\text { desservies }\end{array}$ & $\begin{array}{c}\text { Centrales } \\
\text { thermiques } \\
\text { (refroidissement) }\end{array}$ & Total \\
\hline Eau de surface & 400 & 260 & 50 & 230 & 1090 & 2030 \\
\hline Eau souterraine & 540 & 0 & 200 & 80 & 0 & 820 \\
\hline Total & 940 & 260 & 250 & 310 & 1090 & 2850 \\
\hline$\%$ & 33 & 9 & 9 & 11 & 38 & 100 \\
\hline
\end{tabular}

Tableau 1 - Quantités d'eau prélevées en 1981 par secteur d'utilisation et par origine de l'eau.

\section{Principaux résultats :}

En 1981, les apports d'eau naturels globaux au bassin Loire-Bretagne, formés pour l'essentiel par les précipitations sont estimés à $150 \mathrm{~km}^{3}$ (soit supérieurs de $50 \%$ à ceux estimés en année moyenne à $105 \mathrm{~km}^{3}$ ).

Sur ce total :

- $60 \mathrm{~km}^{3}$, soit $40 \%$, se sont écoulés à la mer dont : $40 \mathrm{~km}^{3}$ en écoulement irrégulier (2/3 de l'écoulement réel total) et $20 \mathrm{~km}^{3}$ en écoulement régulier ( $1 / 3$ de l'écoulement réel total).

Nota : en cette année humide, l'écoulement a été presque le double de celui d'année moyenne : environ $35 \mathrm{~km}^{3}$ dont 27 pour la Loire seule.

$-4.4 \mathrm{~km}^{3}$ soit $2.9 \%$ ont été stockés en nappes souterraines (accroissement des réserves) ou en couverture nivale.

L'ensemble des utilisations aurait :

— prélevé $2.85 \mathrm{~km}^{3}$, soit $4,7 \%$ (et $14 \%$ du flux régulier),

- restitué (aux eaux continentales) $2.5 \mathrm{~km}^{3}$, soit $87.7 \%$ des prélèvements bruts,

- consommé $0.35 \mathrm{~km}^{3}$, soit $12.3 \%$ des prélèvements et $1.7 \%$ du flux écoulé régulier, par évaporation ou déversement en mer,

— régularisé $0.26 \mathrm{~km}^{3}$, soit $0.4 \%$ de l'écoulement total et un accroissement de $1.3 \%$ de l'écoulement régulier naturel,

— épargné $0.12 \mathrm{~km}^{3}$, soit $0.2 \%$ de l'écoulement (solde bénéficiaire des retenues, dont le stock total s'est accru de $14.5 \%)$.

L'évaporation totale, naturelle et induite par les utilisations - notamment l'irrigation -, se serait élevée à $86 \mathrm{~km}^{3}$, soit $57 \%$ des apports, équivalent à une hauteur uniforme de $557 \mathrm{~mm}$.

Les réserves d'eau totales du bassin seraient en moyenne de l'ordre de $130 \mathrm{~km}^{3}$, dont les retenues artificielles forment moins d' $1 \%$, ce qui correspondrait à 3 à 4 années d'écoulement moyen.

La répartition simplifiée des quantités d'eau prélevées en 1981 par secteur d'utilisation et par source d'approvisionnement est indiquée tableau 1 , en $\mathrm{hm}^{3} / \mathrm{an}$ :

L'eau souterraine est ainsi la source d'approvisionnement majeure pour la production d'eau potable et surtout pour l'irrigation ( $80 \%)$, alors que les industries utilisent principalement, et les centrales thermiques exclusivement, l'eau de surface.

Si 1981 avait été une année moyenne, l'indice d'exploitation global des ressources (prélèvements / ressources en eau) aurait été de $8 \%$ et d'environ $22 \%$ en mois d'étiage, ce qui n'est pas négligeable. L'indice de consommation finale (consommations / ressources en eau), plus significatif pour l'ensemble d'un bassin, aurait été de $1 \%$, mais d'environ $6 \%$ en mois d'étiage.

Ultérieurement à l'exercice, des chiffrages révisés des prélèvements, sensiblement supérieurs, ont été opérés par le Ministère de l'environnement (DPP, Direction de l'eau) en 1989 et 1992, sur la base des statistiques de l'Agence de l'eau Loire-Bretagne : les prélèvements totaux se seraient élevés en 1981 à $4.65 \mathrm{~km}^{3}$ (dont 3.1 par les centrales thermiques, ce qui explique l'essentiel de la différence, 0.364 par l'agriculture, 0.305 par l'industrie et 0.882 par les collectivités). Cela aurait porté l'indice d'exploitation global à $7.7 \%$ en 1981 et $13.3 \%$ par rapport aux ressources d'année moyenne. Ces révisions mettent en évidence les incertitudes et les difficultés des chiffrages des prélèvements en eau, données essentielles pour la signification des comptes.

La tendance d'évolution des prélèvements révélée par les statistiques de l'Agence de l'eau de 1981 à 1990 a été une légère croissance jusqu'en $1984\left(5.3 \mathrm{~km}^{3} / \mathrm{an}\right)$ suivie d'une certaine stabilité ou d'une faible décroissance (5.08 en 1988, 4.7 en 1990) traduisant surtout des causes conjoncturelles climatiques.

Toutefois, dans ces statistiques, les prélèvements agricoles, dont la croissance est généralement admise, sont probablement sous-estimés, ce qui doit atténuer la tendance réelle.

\section{IV $\square$ LE COMPTE AFFINÉ, PAR TRIMESTRE, DU HAUT-BASSIN DE LA LOIRE EN 1989-90}

Un compte pilote des eaux continentales sur le haut bassin de la Loire a été réalisé par l'Ifen en 1995. Cette expérience avait pour but de tester la méthode générale d'élaboration des comptes de l'eau " en quantité " sur une échelle 

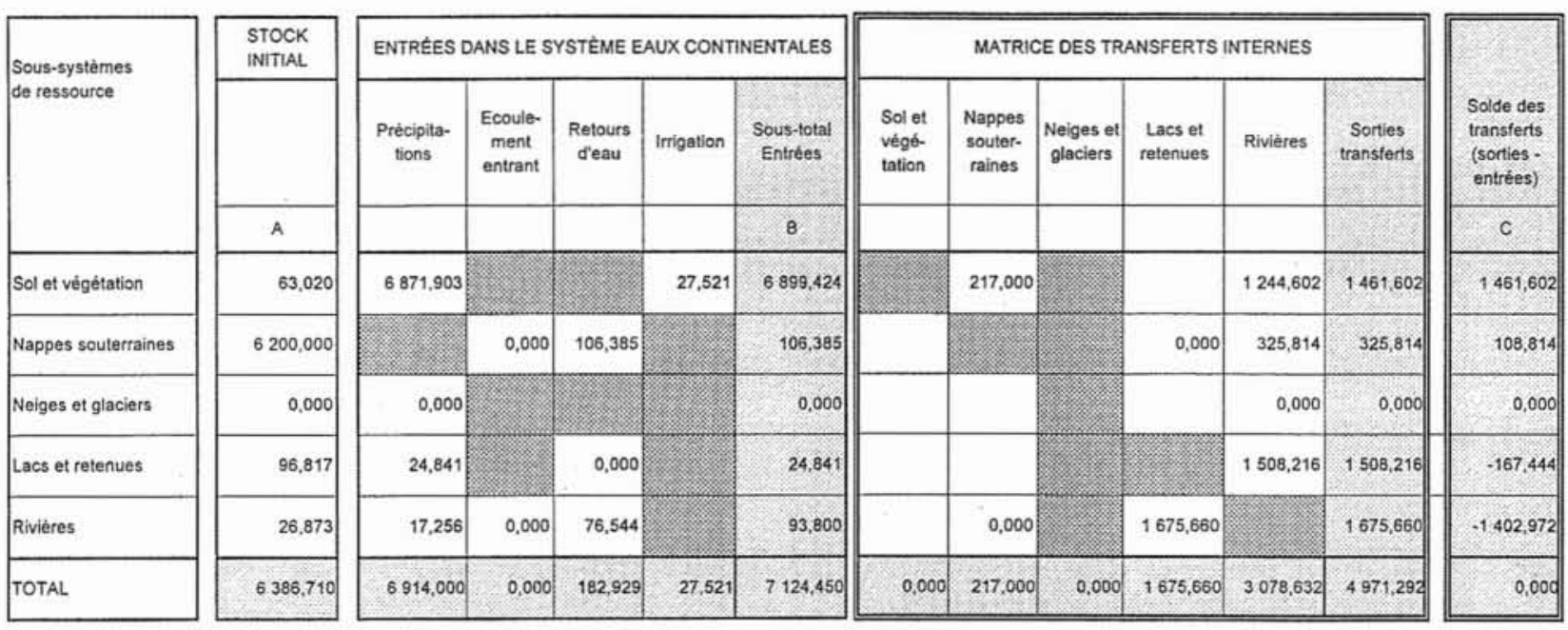

Tableau 2 - Ressources en eau et utilisations dans le haut-bassin de la Loire - année 1989-90 (en millions de $\mathrm{m}^{3}$ ).

spatiale plus petite et sur un pas de temps trimestriel ; d'en évaluer la faisabilité en terme de disponiblité, de qualité, d'efforts de collecte et de traitement des données utiles à la construction des comptes - dont la structure a été un peu révisée par rapport à celle des premiers tableaux comptables.

Quatre comptes trimestriels (et le total annuel) ont été établis pour l'année hydrologique 1989-1990, sur un bassin versant d'une superficie de $9315 \mathrm{~km}^{2}$. Ce bassin est du même ordre de grandeur (légèrement plus grand) que le bassin RNDE n 0401, dénommé " bassin de la Loire à l'amont de Roanne ". Le fait d'avoir choisi une année sèche (moins de $50 \%$ de la moyenne pluriannuelle de l'écoulement) rend d'autant plus visible la pression des prélèvements sur la ressource en eau, et fournit un bon exemple d'analyse des situations les plus tendues.

\section{Principaux résultats - Le compte annuel :}

Deux tableaux comptables sont présentés ici. Ils décrivent tous les mouvements d'eau du bassin de la Haute-Loire d'octobre 1989 à septembre 1990 : à la fois les entrées/sorties et les mouvements de stocks, et à la fois les échanges naturels et ceux provoqués par l'homme (tableaux 2 et 3 ).

En 1989-90, les apports d'eau naturels globaux sur le bassin de la Haute-Loire, formés pour l'essentiel par les précipitations, sont estimés à $6.9 \mathrm{~km}^{3}$, soit à $742 \mathrm{~mm} / \mathrm{an}$, en hauteur moyenne surfacique.

Sur ce total :

$-1.4 \mathrm{~km}^{3}$, soit $20 \%$, se sont écoulés dans le bassin aval, dont : $0.3 \mathrm{~km}^{3}$ en écoulement régulier (20\% de l'écoulement réel total), $1.1 \mathrm{~km}^{3}$ en écoulement irrégulier ( $80 \%$ de l'écoulement réel total)

$-5.5 \mathrm{~km}^{3}$, soit $80 \%$, se sont évaporés dans l'atmosphère.

L'ensemble des utilisations aurait :

- prélevé plus de $0.35 \mathrm{~km}^{3}$, soit environ $21 \%$ du débit mesuré (et plus de $100 \%$ du flux écoulé régulier),

\begin{tabular}{|c|c|c|c|c|c|}
\hline \multirow{3}{*}{$\begin{array}{l}\text { Sous-systèmes } \\
\text { de ressource }\end{array}$} & \multicolumn{4}{|c|}{$\begin{array}{l}\text { SORTIES DU SYSTÈME EAUX } \\
\text { CONTINENTALES }\end{array}$} & $\begin{array}{l}\text { STOCK } \\
\text { FINAL }\end{array}$ \\
\hline & $\begin{array}{l}\text { Prélève- } \\
\text { ments }\end{array}$ & $\begin{array}{l}\text { Evapo- } \\
\text { transpi- } \\
\text { ration }\end{array}$ & $\begin{array}{c}\text { Ecoule- } \\
\text { ment } \\
\text { sortant } \\
\text { (extérieur) }\end{array}$ & $\begin{array}{l}\text { Sous-total } \\
\text { Sorties }\end{array}$ & \\
\hline & & & & D & $A+B-C-D$ \\
\hline Sol et végétation & & 5437.822 & & 5437,822 & 63,020 \\
\hline Nappes souterraines & 38,850 & & 0,600 & 39,450 & 6158,121 \\
\hline Neiges et glaciers & & 0.000 & & 0,000 & 0,000 \\
\hline Lacs et retenues & 217,280 & 27,394 & & 244,674 & 44,428 \\
\hline Rivières & 95,897 & 28,891 & 1381,675 & 1506,463 & 17,182 \\
\hline TOTAL & 352,027 & 5494,107 & 1382,275 & 7228,409 & 6282,751 \\
\hline
\end{tabular}

- restitué (aux eaux continentales) $0.18 \mathrm{~km}^{3}$, soit $51 \%$ des prélèvements bruts,

- consommé $0.17 \mathrm{~km}^{3}$, soit $49 \%$ des prélèvements et $56 \%$ du flux écoulé régulier, par évaporation et exportations d'eau. Il y a là une originalité du bassin : l'existence d'une dérivation d'eau vers l'extérieur par le système hydroélectrique de Montpezat, qui a prélevé cette année-là $0.1 \mathrm{~km}^{3}$, soit $7.5 \%$ du débit de la Loire, ou encore $87 \%$ du seul bassin supérieur correspondant à l'aménagement du système Montpezat,

- emprunté $0.1 \mathrm{~km}^{3}$, (le stock total des retenues a diminué de $54 \%$ ).

La répartition des quantités d'eau prélevées en 1989-90, par secteur d'utilisation et par source d'approvisionnement, peut se visualiser de la manière suivante (figure 1). 


\begin{tabular}{|c|c|c|c|c|c|c|c|}
\hline \multirow{3}{*}{\multicolumn{2}{|c|}{$\begin{array}{l}\text { Total année } \\
\text { milliers m3 }\end{array}$}} & \multicolumn{6}{|c|}{ ENTREEES DANS LE SYSTEMME DUTILISATION } \\
\hline & & \multicolumn{2}{|c|}{ Prélèvements } & \multirow[t]{2}{*}{$\begin{array}{l}\text { Impor- } \\
\text { tations }\end{array}$} & \multirow[t]{2}{*}{$\begin{array}{c}\text { Sous-lotal } \\
\text { Entrees }\end{array}$} & \multirow[t]{2}{*}{$\begin{array}{l}\text { Entrées } \\
\text { Echanges }\end{array}$} & \multirow[t]{2}{*}{$\begin{array}{l}\text { Total } \\
\text { Entrées }\end{array}$} \\
\hline & & eaux supert & eaux sout & & & & \\
\hline $\begin{array}{l}\text { Prod-distrib } \\
\text { d'eau } \\
\text { potable }\end{array}$ & & 81918,40 & 23187,60 & & 105106,00 & $5.837,00$ & 110943,00 \\
\hline $\begin{array}{l}\text { Prod-distrib } \\
\text { d'eau } \\
\text { dirrigation }\end{array}$ & & 42262,00 & & & 42262,00 & 0,00 & 42262,00 \\
\hline $\begin{array}{l}\text { Entreprisos } \\
\text { Industrielles }\end{array}$ & & 4932,80 & 3176,80 & & 8109,80 & 12433,00 & 20.542 .60 \\
\hline $\begin{array}{l}\text { Producteurs } \\
\text { o'electricité }\end{array}$ & & 113294,00 & & & 113294,00 & 0,00 & 113294,00 \\
\hline \multirow[t]{3}{*}{ Agriculteurs } & Imigants & 4972,00 & 2486,00 & & 7458,00 & 23121,00 & 30579,00 \\
\hline & eleveurs & 2725,60 & 10000,00 & & 12725,60 & $1938: 00$ & 14663,60 \\
\hline & Total & 7697.60 & \multicolumn{2}{|l|}{12486,00} & 20183,60 & 25059,00 & 45242,60 \\
\hline Ménages & & & & & 0,00 & 44925,00 & 44926,00 \\
\hline Services pub & Asst & & & & 0,00 & 51734,36 & 51734,36 \\
\hline & Autres & 63072.00 & & & 63072,00 & 16958,00 & 80030.00 \\
\hline TOTAL & & 313176,80 & 38850,40 & 0,00 & 352027,20 & 156947,36 & 508974,56 \\
\hline
\end{tabular}

\begin{tabular}{|c|c|c|c|c|c|c|}
\hline \multicolumn{7}{|c|}{ SORTIES DU SYSTÉME DUTILISATION } \\
\hline \multicolumn{3}{|c|}{ Retours creau a lintérieur } & \multirow{2}{*}{$\begin{array}{c}\text { Consom- } \\
\text { mation nette } \\
\text { (Evapo.) }\end{array}$} & \multirow{2}{*}{\begin{tabular}{|c|} 
Sorties \\
deaut a \\
rexterieur \\
(export.)
\end{tabular}} & \multirow{2}{*}{$\begin{array}{l}\text { Sous-total } \\
\text { Sorties }\end{array}$} & \multirow[t]{2}{*}{ Total Sorties } \\
\hline $\begin{array}{l}\text { Pertes et } \\
\text { fultes }\end{array}$ & $\begin{array}{l}\text { Rejets } \\
\text { directs }\end{array}$ & $\begin{array}{l}\text { inflitration } \\
\text { inrigation }\end{array}$ & & & & \\
\hline 24442,00 & 4409,00 & & & & $28,851,00$ & $110,943,00$ \\
\hline 15768,00 & 3154,00 & & 219,00 & & 19141,00 & 42262.00 \\
\hline 2054,24 & 4108,48 & & 3081,44 & & 9244,16 & 20542,60 \\
\hline & & & 10010,00 & 103284,00 & 113294,00 & 113294,00 \\
\hline 3057,90 & & 11008,44 & 16512.66 & & 30579,00 & 30579,00 \\
\hline & & & 14663.60 & & 14663,60 & 14663,80 \\
\hline 3057.90 & & 11008,44 & 31176,26 & & 45242,60 & 45242,60 \\
\hline 8985,20 & & & 4492,60 & & 13477,80 & 44928,00 \\
\hline 5173,44 & 46560,92 & & & & 51734,36 & 51734,36 \\
\hline 35896,40 & 18311,72 & & 1066,16 & 15768,00 & 71042.28 & $80,030,00$ \\
\hline 95377,18 & 76544,12 & 11008,44 & 50045,46 & 119052,00 & 352027,20 & 508974.56 \\
\hline
\end{tabular}

Tableau 3 - Utilisations de la ressource en eau dans le haut-bassin de la Loire - année 1989-90 (en milliers de $\mathrm{m}^{3}$ ).

\begin{tabular}{|c|c|c|c|c|c|c|c|c|c|c|}
\hline \multirow{3}{*}{\multicolumn{2}{|c|}{$\begin{array}{l}\text { Total année } \\
\text { milliers m33 }\end{array}$}} & \multicolumn{9}{|c|}{ MATRICE DES ĖCHANGES ENTRE AGENTS } \\
\hline & & \multirow[t]{2}{*}{$\begin{array}{l}\text { Prod. } \\
\text { distrib } \\
\text { deau } \\
\text { potable }\end{array}$} & \multirow[t]{2}{*}{$\begin{array}{c}\text { Prod- } \\
\text { distrib } \\
\text { d'eau } \\
\text { dirimigation }\end{array}$} & \multirow[t]{2}{*}{$\begin{array}{c}\text { Entre-prises } \\
\text { indus- } \\
\text { trielles }\end{array}$} & \multirow[t]{2}{*}{$\begin{array}{l}\text { Prod } \\
\text { o'telec. } \\
\text { tricití }\end{array}$} & \multirow[t]{2}{*}{$\begin{array}{c}\text { Agricul. } \\
\text { teurs }\end{array}$} & \multirow[t]{2}{*}{ Mènages } & \multicolumn{2}{|c|}{ Services publics } & \multirow[t]{2}{*}{$\begin{array}{l}\text { Sous total } \\
\text { Sorties } \\
\text { Echanges }\end{array}$} \\
\hline & & & & & & & & Asst & Autres & \\
\hline $\begin{array}{l}\text { Prod-distrib } \\
\text { deau } \\
\text { potable }\end{array}$ & & 5837,00 & & 12433,00 & & 1938,00 & 44926,00 & & 16958,00 & 82.092 .00 \\
\hline $\begin{array}{l}\text { Prod-distrib } \\
\text { deau } \\
\text { dilirigation }\end{array}$ & & & & & & 23121,00 & & & & 23121,00 \\
\hline $\begin{array}{l}\text { Entreprises } \\
\text { industrinelles }\end{array}$ & & & & & & & & 11298.44 & & 11298,44 \\
\hline $\begin{array}{l}\text { Producteurs } \\
\text { ótelectricté }\end{array}$ & & & & & & & & & & 0.00 \\
\hline Agriculteurs & irrigants & & & & & & & & & 0.00 \\
\hline & eleveur: & & & & & & & & & 0,00 \\
\hline & Total & & & & & & & & & 0,00 \\
\hline Mènages & & & & & & & & 31448,20 & & $31,448.20$ \\
\hline Services pub & b Asst & & & & & & & & & 0.00 \\
\hline & Autres & & & & & & & 8987,72 & & 8.987 .72 \\
\hline TOTAL & & $5.837,00$ & 0.00 & 12433.00 & 0,00 & 25059,00 & 44926.00 & $|51734,36|$ & $16.958,00$ & 156.947 .36 \\
\hline
\end{tabular}




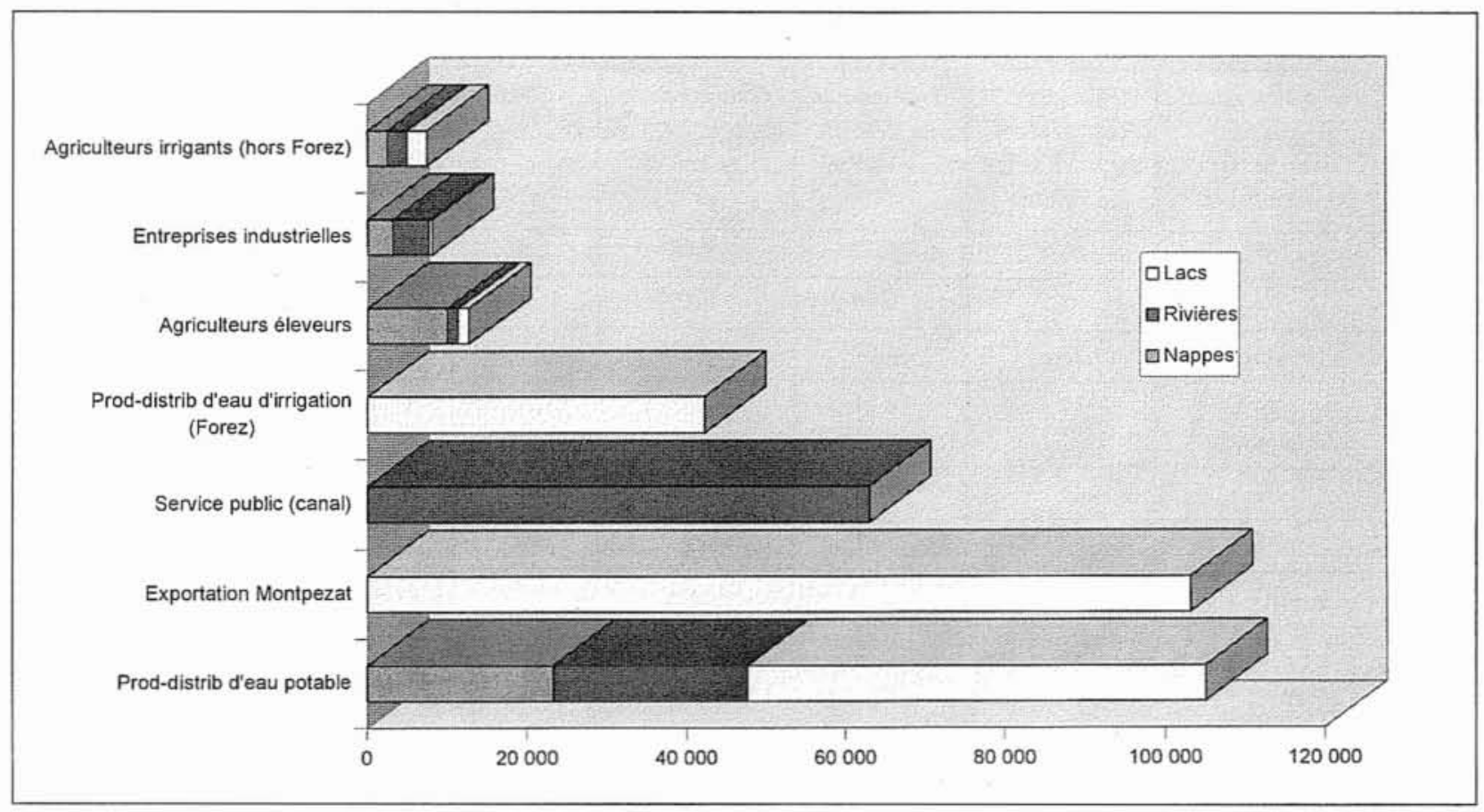

1. Répartition des prélèvements annuels en eau par agent et par sous-système de ressource (en milliers de m $^{3}$ ).

En pourcentage du total annuel, la répartition des prélèvements peut aussi se représenter de la façon suivante (fig. 2) :

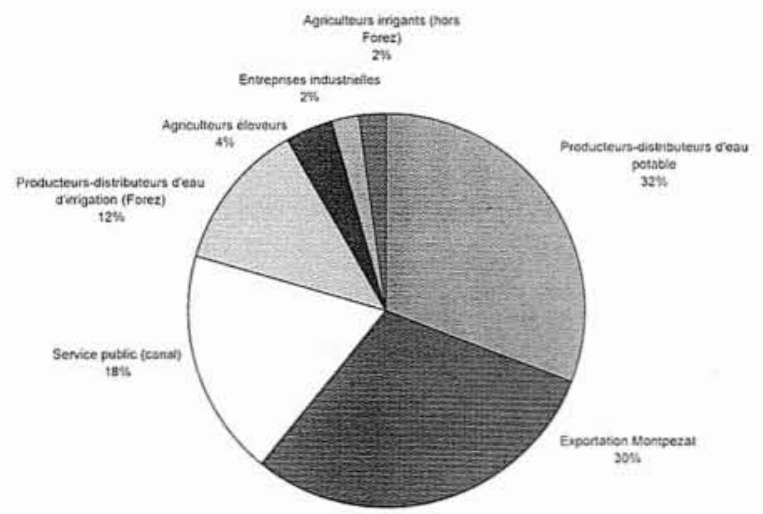

2. Répartition des prélèvements annuels en eau par agent (en \%).

\section{Principaux résultats - Les comptes trimestriels :}

- La saisonnalité du compte a permis d'affiner l'analyse des utilisations de l'eau. Elle a notamment permis de montrer que d'un point de vue comptable, si les volumes prélevés étaient totalement consommés (sans retours) et si les barrages n'existaient pas, les prélèvements en eau n'auraient pas été réalisables cette année-là dans le haut-bassin de la Loire : globalement, la somme des prélèvements annuels en eau est supérieure au débit d'étiage naturel (ou phase régulière de l'écoulement) pour l'année 1989-90. L'écart

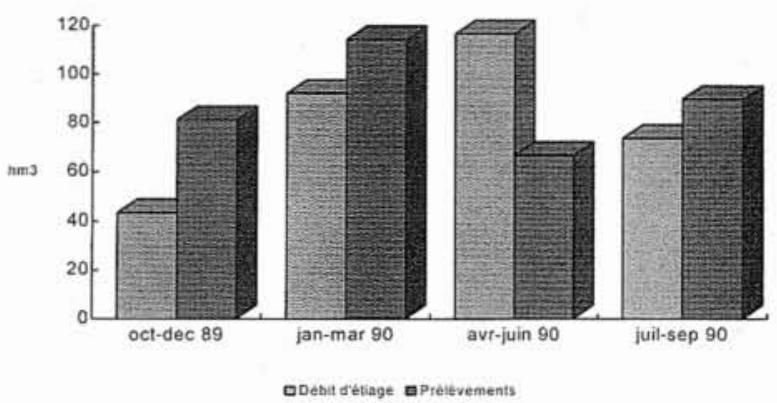

3. Comparaison, par trimestre, de la part régulière de la ressource (débits d'étiage) et des prélèvements $\left(\mathrm{en} \mathrm{hm}^{3}\right)$.

s'amplifie dans le trimestre le plus pauvre en eau (octobre décembre 89 ), où la somme des prélèvements est presque le double de l'écoulement régulier naturel ( 81 millions de $\mathrm{m}^{3}$ pour 43 millions de $\mathrm{m}^{3}$ ) (figure 3 ).

- Cette situation n'a été possible que grâce aux retours d'eau des activités utilisatrices d'eau et aux soutiens d'étiage par les lâchures de barrages. Elle montre en particulier le rôle actif joué par la régulation des barrages en Loire amont, sur les possibilités de prélèvements en période de basses eaux.

— La variabilité des prélèvements entre les trimestres n'est pas négligeable, notamment entre le deuxième (janvier-mars 1990) et le troisième (avril-juin 1990) trimestre du compte (figure 3). Elle est à imputer aux deux secteurs d'utilisation dont les demandes en eau sont les plus variables selon les tri- 
Tableau 4 - Indices d'exploitation et de consommation par bassin en 1990.

\begin{tabular}{|l|c|c|c|c|c|c|c|c|}
\hline Bassin & $\begin{array}{c}\text { Flux moyen annuel } \\
\text { de ressource } \\
\text { naturelle }(\mathrm{Q}) \\
\text { en } \mathrm{km}^{3} / \mathrm{an}\end{array}$ & $\begin{array}{c}\text { Flux année } \\
\text { décennale sèche } \\
\left(\mathrm{Q}_{10}=2 / 3 \text { de } \mathrm{Q}\right) \\
\text { en km/3an }\end{array}$ & $\begin{array}{c}\text { Prélèvements } \\
\text { en } 1990(\mathrm{P}) \\
\text { en } \mathrm{km}^{3} / \mathrm{an}\end{array}$ & $\begin{array}{c}\text { Consommations } \\
\text { nettes en } 1990(\mathrm{C}) \\
\text { en } \mathrm{km}^{3} / \mathrm{an} \\
\text { en } \%\end{array}$ & $\begin{array}{c}\text { Indice d'exploitation } \\
1990 \\
\text { en } \%\end{array}$ & \multicolumn{2}{|c|}{$\begin{array}{c}\text { Indice de } \\
\text { consommation } 1990 \\
\text { en \% }\end{array}$} \\
\hline AG & 40 & 26.5 & 2.57 & 0.88 & 6.4 & 9.7 & 2.2 & 3.3 \\
\hline AP & 4 & 2.5 & 0.66 & 0.15 & 16.5 & 26.4 & 3.7 & 6.0 \\
\hline LB & 35 & 23 & 4.67 & 0.92 & 13.3 & 20.3 & 2.6 & 4.0 \\
\hline RM & 14 & 10 & 8.71 & 0.43 & 62.2 & 87 & 3.1 & 4.3 \\
\hline RMC & 70 & 46 & 17.31 & 2.41 & 24.7 & 37.6 & 3.4 & 5.2 \\
\hline SN & 18 & 12 & 3.81 & 0.9 & 21.2 & 31.7 & 5 & 7.5 \\
\hline Total & 181 & 120 & 37.73 & 5.69 & 20.8 & 31.4 & 3.1 & 4.7 \\
\hline
\end{tabular}

Sources: "L'eau en France : nature et ressources", MARGAT \& TRUCHOT, Annales des Mines, juillet-août 1988.

"Les prélèvements et consommations d'eau en France de 1981 à 1990 ", Ministêre de l'Environnement, Direction de I'Eau, juillet 1992.

mestres : prélèvements hydroélectriques de Montpezat et prélèvements agricoles. Dans le premier cas (hydroélectricité), le trimestre $\mathrm{n}^{\circ} 2$ (janvier-mars 90 ) totalise presque la moitié des prélèvements annuels, et, dans le second cas (agriculture irriguée), plus de la moitié des prélèvements annuels est réalisé au cours du trimestre $n^{\circ} 4$ (juillet-septembre 90 ) (figure 4 ).

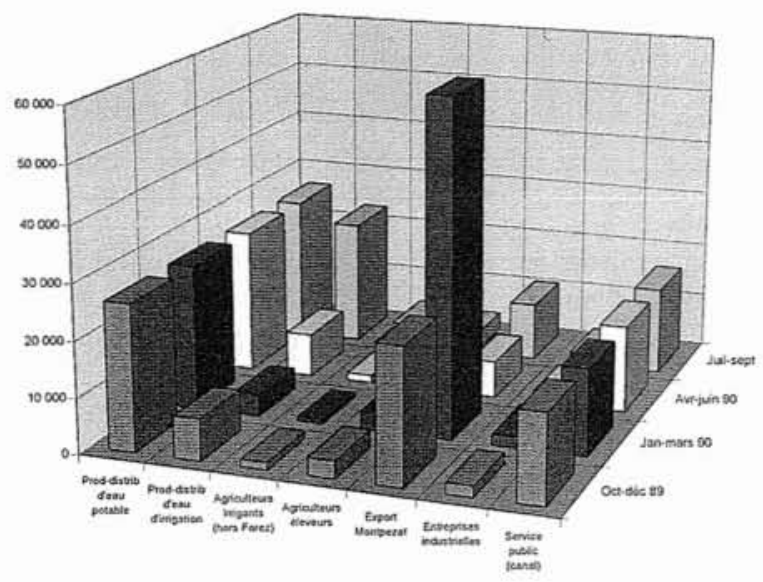

Figure 4 : Répartition trimestrielle des prélèvements par agent (en milliers de $\mathrm{m}^{3}$ )

\section{$\mathrm{V}$ GONCLUSION}

Le bassin de la Loire, en 1990, offre $19.3 \%$ des ressources en eau moyennes de la France et il est le siège de $12.4 \%$ des prélèvements totaux.
Ce bassin se trouve au 5ème rang des bassins français (dans l'ordre décroissant) par ses indices moyens d'exploitation et de consommation (tableau 4).

Par rapport à la ressource d'année moyenne de tout le bassin Loire-Bretagne, on a prélevé en $199013.3 \%$ et consommé (consommation nette) $2.6 \%$. Par rapport à la ressource d'année décennale sèche, ces indicateurs sont respectivement de $20.3 \%$ et $4 \%$ (tableau 4 ).

Même si l'étude du bassin Loire Bretagne pour une année donnée permet diverses comparaisons entre les bassins, elle reste cependant trop globale pour permettre une analyse affinée à la fois dans l'espace et dans le temps.

Comme l'a montré l'expérience pilote menée sur le haut bassin de la Loire, une plus grande précision est possible : les " bassins RNDE", dont les étendues sont du même ordre de grandeur, paraissent être les cadres pertinents pour une analyse localisée des pressions besoins/ressources, de même que la trimestrialisation a mis en évidence des situations qui n'apparaissaient pas dans le compte annuel.

En d'autres termes, l'analyse globale ne fait pas apparaître de tension significative entre les besoins et les ressources en eau, alors que dans certains cadres " régionaux" et selon les saisons il peut y avoir des tensions non négligeables sur le plan strictement quantitatif - ce qui peut avoir des conséquences sur le plan qualitatif.

\section{Bibliographie}

[1] COLLECTIF (1986). Les comptes du patrimoine naturel (INSEE, les collections de l'INSEE 137-138 C, $\mathrm{n}^{\circ}$ 535-536, Déc. 552, p. Paris) - Chap. 5 -, J. Margat, Le compte des eaux continentales

[2] BABILLOT Pascale (1995) - Compte de l'eau sur le bassin de la haute Loire (IFEN, Juil. 126 p., Orléans) 